\title{
Effect of pressure on the phase behavior and structure of water confined between nanoscale hydrophobic and hydrophilic plates
}

\author{
Nicolas Giovambattista ${ }^{1}$, Peter J. Rossky ${ }^{2}$, and Pablo G. Debenedetti ${ }^{1}$ \\ 1 Department of Chemical Engineering, \\ Princeton University, Princeton, NJ 08544-5263 USA \\ 2 Department of Chemistry and Biochemistry, \\ Institute for Theoretical Chemistry, \\ University of Texas at Austin, \\ Austin, Texas 78712 USA
}

(Dated: 17 Feb. 2006)

\begin{abstract}
We perform systematic molecular dynamics simulations of water confined between two nanoscale plates at $T=300 \mathrm{~K}$. We investigate the effect of pressure $(-0.15 \mathrm{GPa} \leq P \leq 0.2 \mathrm{GPa})$ and plate separation $(0.4 \mathrm{~nm} \leq d \leq 1.6 \mathrm{~nm})$ on the phase behavior of water when the plates are either hydrophobic or hydrophilic. When water is confined between hydrophobic plates, capillary evaporation occurs between the plates at low enough $P$. The threshold value of $d$ at which this transition occurs decreases with $P$ (e.g., $1.6 \mathrm{~nm}$ at $P \approx-0.05 \mathrm{GPa} ; 0.5 \mathrm{~nm}$ at $P \approx 0.1 \mathrm{GPa}$ ), until, at high $P$, no capillary evaporation occurs. For $d \approx 0.6 \mathrm{~nm}$ and $P \geq 0.1 \mathrm{GPa}$, the system crystallizes into a bilayer ice. A $P-d$ phase diagram showing the vapor, liquid, and bilayer ice phases is proposed. When water is confined by hydrophilic (hydroxylated silica) plates, it remains in the liquid phase at all $P$ and $d$ studied. Interestingly, we observe, for this case, that even at the $P$ at which bulk water cavitates, the confined water remains in the liquid state. We also study systematically the state of hydration at different $P$ for both kinds of plates. For the range of conditions studied here, we find that in the presence of hydrophobic plates the effect of $P$ is to enhance water structure and to push water molecules toward the plates. The average orientation of water molecules next to the hydrophobic plates does not change upon pressurization. In contrast, in the presence of hydrophilic plates, water structure is insensitive to $P$. Hence, our results suggest that upon pressurization, hydrophobic plates behave as 'soft' surfaces (in the sense of accommodating pressure-dependent changes in water structure) while hydrophilic walls behave as 'hard' surfaces.
\end{abstract}

PACS numbers:

\section{INTRODUCTION}

Confining geometries that contain thin water films are common in biology, geology and engineering. Examples include ion channels [1, 2, 3, 4, 5, 6], mesoscopic surfactant assemblies [7, 8], mineral inclusions 9], zeolites [10], and microfluidic technologies [11]. Understanding the changes in water structure, dynamics and thermodynamics due to interactions with confining surfaces is therefore of interest in a wide variety of scientific problems and technical applications, such as the inhibition of corrosion 12], heterogeneous catalysis, the design of superhydrophobic surfaces 13], the ascent of sap in plants 9], the function of biological membranes 14|, and the development of 'lab on a chip' technologies [15].

The behavior of water near hydrophobic surfaces has attracted considerable attention, both on account of the intrinsic scientific interest of the thermodynamic problem (e.g., 16, 17, 18, 19, 20, 21, 22, 23, 24, 25, 26]), as well as its relevance to water permeation through membrane channels 2, 3, 4, 5, 6, 27 and carbon nanotubes [28, 29], micelle formation [20, 30], and the amphiphaticity of membrane proteins [14, 31, 32]. Hydrophobic interactions are also important in phase transfer catalysis [33], chemical self-assembly of macroscopic objects 34], and capillary evaporation [35, 36, 37].

More than thirty years ago, Stillinger 23] argued that when a non-polar solute is sufficiently large, the watersolute interface resembles that between water and its own vapor. Lum et al. 21] proposed a theoretical approach to describe the crossover between the solvation of small and large hydrophobic objects. Bulk thermodynamics and entropic contributions to the free energy of solvation are dominant in the former case. Interfacial thermodynamics and enthalpic contributions to solvation free energy play a key role in the latter case. The crossover length scale between these two regimes, $\approx 1 \mathrm{~nm}$ at ambient conditions, is a sensitive function of pressure and the presence of co-solutes [38]. From a microscopic point of view, this length scale is related to the different manner in which water molecules arrange around solutes of different sizes. In the presence of small hydrophobic solutes, water molecules rearrange in such a way that the solutes can be accommodated into water's hydrogen-bond (HB) network. Thus, small hydrophobic solutes are caged in clathrate-like structures 39, 40, 41] with $\mathrm{HB}$ vectors (defined as the four tetrahedral vectors pointing outward from the oxygen atom of each water molecule along the two oxygen-hydrogen bond donor, and the two "lone pair electrons" acceptor directions) avoiding pointing toward the non-polar solute. As the solute size increases (to $\approx 1 \mathrm{~nm}$ at ambient conditions) 42, 43, 44, 45], such clathrate-like structure cannot be maintained: HBs break, inducing a restruc- 
turing of the HB network. In this case, the hydration structure shows an orientational inversion with respect to the clathrate-like structure, with HBs pointing toward the solute [42, 43, 46, 47, 48].

When two hydrophobic surfaces approach each other, a confined water film can be thermodynamically destabilized with respect to the vapor at small enough separations [21, 24, 35, 36, 49]. A plausible interpretation of the experimentally observed long-ranged attractions between hydrophobic surfaces (e.g., $10-100 \mathrm{~nm}$ ) 50, 51, 52, 53. involves dewetting of the inter-plate region. As shown by Bérard et al. 54], such dewetting is not particular to water, but arises as a general consequence of weak fluidwall interactions relative to fluid-fluid interactions [24]. The generality of confinement-induced drying notwithstanding, interest in this phenomenon has focused predominantly on the case in which the confined fluid is water and the confining surfaces are hydrophobic. Several computational studies of this situation exist (e.g., [36, 44, 55, 56, 57, 58, 59, 60, 61)).

Kauzmann's influential review 62 first pointed out the importance of hydrophobic interactions in protein folding. It is generally accepted that the formation of a core of hydrophobic residues from which water is partially excluded is a dominant force in the folding of globular proteins [62, 63, 64, 65, 66, 67, 68, 69, 70, 71]. However, the surface of a protein is a geometrically, chemically, and electrically heterogeneous object, and the distribution, structure, and dynamics of water near such a surface is expected to be quite different from that found near idealized surfaces, such as perfectly hydrophobic walls. In fact, computer simulations have clearly shown a range of water behavior in response to surface heterogeneity. Cheng and Rossky 72] studied the hydration of the polypeptide melittin. They found that clathrate-like structures dominate near convex surface patches, while near flat regions the hydration shell fluctuates between clathrate-like and less ordered (inverted) structures. The collapse of a two-domain BphC enzyme showed no dewetting in the inter-domain region when the units were brought together down to a distance of $0.4 \mathrm{~nm}$ [73]. A dewetting transition was observed only when electrostatic interactions were turned off 73]. In contrast, dewetting has been observed in the collapse of of the melittin tetramer 74]; however, even single mutations were shown to prevent drying.

The above protein folding examples illustrate the importance of understanding water behavior near complex, heterogeneous surfaces. Materials science and engineering applications involving chemically nanopatterned substrates (e.g., 75]) likewise will require an understanding of water structure near heterogeneous interfaces. Molecular simulations should prove extremely powerful in addressing this problem, because they allow exquisitely sensitive control of surface geometry and chemistry. Questions such as the manner in which the characteristic length of hydrophobic patches and the pattern of their distribution on a hydrophilic surface affect confined wa- ter structure and dynamics, or the effect of surface chemistry in promoting or preventing bulk cavitation following dewetting of a confined region are ideally suited for computational scrutiny. The recent work of Koishi et al. [59] is an excellent example of the valuable insights that can be obtained by molecular simulations regarding water behavior near complex surfaces.

This paper is the first report of an ongoing computational investigation aimed at understanding the influence of surface heterogeneity and bulk thermodynamic conditions (pressure, temperature) on the structure, dynamics, and thermodynamics of confined water. Here we establish the 'base case' conditions by comparing water structure and phase behavior at $300 \mathrm{~K}$ when confined by purely hydrophobic and purely hydrophilic nanoscale surfaces $(0.4 \leq d \leq 1.6 \mathrm{~nm})$. Li et al. [58] have recently studied hydration and dewetting near hydrophilic and hydrophobic plates (but with different chemistry than the ones we consider here), at a single pressure. An important aspect of the present work is that we perform such a comparison over a broad range of pressures $(-0.15 \leq P \leq 0.2 \mathrm{GPa})$. In subsequent papers we will report results on water confined between heterogeneous plates with engineered 'patchiness'.

Our computational study should be placed in the context of a considerable body of experimental work aimed at studying systematically the properties of water confined by hydrophobic and hydrophilic surfaces. The forces between hydrophobic surfaces in aqueous solution have been measured with the surface force apparatus (e.g., 50, 51, 52, 53, 76]). Such studies revealed the existence of long-range attractions between hydrophobic surfaces, over distances of the order of $100 \mathrm{~nm}$, and investigated the origin of this phenomenon. Bridging cavities and microbubbles were linked in several [50, 52, 53], but not all [51] of these studies, to the long-ranged attraction. Zhang et al. 77] investigated the response to shear stresses of water confined between adjoining surfaces, one hydrophobic and the other hydrophilic (Janus surface). They found that the competing effects of these surfaces gave rise to unusually noisy responses to shear. Jensen et al. 55. used X-ray reflectivity to study the contact region between water and an extended paraffin surface. They found that drying was confined to a very narrow $(\approx 1.5 \mathrm{~nm})$ region. Ruan et al. $[78$ investigated the structure and dynamics of water near a hydrophilic surface using ultrafast electron crystallography. They observed coexistence between ordered surface water (up to $1 \mathrm{~nm}$ thick) and crystalline islands. Reference to particular aspects of the studies summarized above will be made throughout this paper, stressing, whenever possible, the relationship between experimental observations and our calculations.

This paper is organized as follows. In the next section we describe the simulation details. The results for hydrophobic plates are presented in Sec. III calculations on water confined by hydrophilic plates are reported in Sec. IV We summarize our main conclusions in Sec. D 


\section{SIMULATION DETAILS}

We perform molecular dynamics (MD) simulations in the NPT ensemble. The system is composed of $N=3375$ water molecules in which are inmersed two identical finite three dimensional nanoscale plates. The temperature is fixed at $T=300 \mathrm{~K}$ by using a Berendsen thermostat [79]. The pressure ranges from $P=-0.15 \mathrm{GPa}$ to $P=$ $0.2 \mathrm{GPa}$, and is controlled by coupling the system volume to an external bath at $P$ (analogous to the Berendsen thermostat 79 ).

We simulate a cubic sample using periodic boundary conditions along the three directions. Water molecules are modelled with the SPC/E pair potential [80. The plates are introduced symmetrically about the center of the box such that they are parallel to the $x-y$ plane and equidistant from the $z=0$ plane. The plate dimensions $\left(3.215 \times 3.217 \times 0.866 \mathrm{~nm}^{3}\right)$ are smaller than the box size (which varies with $P$ but always exceeds $4.85 \mathrm{~nm}$ under the conditions investigated here). Figures 1(a) and (b) show a fully hydroxylated silica plate (one of the two kinds of plates used in this study) and Fig. 1(c) is a cross section, showing the water molecules and the two cavities where the plates are located. The plates are fixed in space throughout.

We consider two kinds of plates: hydrophobic or hydrophilic. Their common underlying structure corresponds to four layers of $\mathrm{SiO}_{2}$ [see Fig. 1(b)] reproducing the (1.1.1) octahedral face of cristobalite [81, 82] [see Fig. 1(a)]. The unit cell of $\mathrm{SiO}_{2}$ is idealized as a perfect tetrahedron with $O-O$ and $S i-O$ distances of $0.247 \mathrm{~nm}$ and $0.151 \mathrm{~nm}$, respectively. The hydrophobic plates consist of the above-described structure; however, the ' $\mathrm{Si}$ ' and ' $\mathrm{O}$ ' atoms interact with water molecule $O$ atoms exclusively via a Lennard-Jones potential ( $\epsilon$ and $\sigma$ parameters are given in Table 【). Each hydrophobic plate is composed of 674 atoms.

The hydrophilic plates correspond to fully hydroxylated silica and are obtained by attaching a hydrogen atom to each surface oxygen atom on the four plate surfaces [see Figs. [(d) and (e)]. The $O-H$ distance is chosen to be the same as in the SPC/E model, i.e. 0.1 $\mathrm{nm}$. The $S i$ and $O$ atoms are located in fixed positions (as in the hydrophobic plates) but the $H$ atoms on the surface are able to move with fixed bond lengths and bond angles; each $H$ atom can reorient in a circle. Such circular motion occurs in a plane parallel to the plate at a distance $0.033 \mathrm{~nm}$ away from the $O$ atom plane of the $\mathrm{Si}-\mathrm{O}-\mathrm{H}$ groups. The resulting $\mathrm{Si}-\mathrm{O}-\mathrm{H}$ angle is $109^{\circ} .27$ [see Fig. [(e)]. Each hydrophilic plate is composed of 778 atoms. Only the polar $\mathrm{Si}-\mathrm{O}-\mathrm{H}$ groups at the surface carry partial electric charge [charge values are given in Table प. Electrostatic interactions are treated using the Ewald sum method with a cutoff distance of $0.79 \mathrm{~nm}$ and parameters $m_{\max }=5^{3}$ (for the number of vectors in the reciprocal-space sum) and $\alpha=0.4$ (for the width of the screening-charge Gaussian distribution) [83, 84].
The vibrations of the $S i$ and $O$ atoms have not been taken into account in this work. A computational study of water in single-wall carbon nanotubes indicates that flexibility can affect a channel's apparent hydrophobicity 29]. On the other hand, a molecular dynamics study of water droplets on graphite revealed a negligible effect of substrate vibrations on the contact angle [85]. The lattice constant for the cristobalite structure used in this work is $0.494 \mathrm{~nm}$. Invoking a Lindemann-type estimate, the amplitude of individual atomic vibrations should be considerably smaller than the melting threshold (10\% of the lattice constant), i.e. $0.05 \mathrm{~nm}$ in our case. This upper bound on the magnitude of substrate atomic vibrations is an order of magnitude smaller than the closest interplate separation considered in our work. Thus, while the effect of crystal vibrations on the structure and dynamics of confined water deserves careful attention and will be explored in future studies, we believe that the present rigid wall base case is a reasonable starting point for our investigations.

The pressure is calculated using the virial expression, taking into account the fact that some atoms in the plates are fixed and others can move [84, 86]. We also use the link cell and neighbor list methods to calculate the pair interactions [87].

We perform simulations for different time intervals depending on the kind of plates and their separation. Simulation times are indicated in Table $\amalg$ One of the reasons we chose these simulation times is that when there is no capillary evaporation or crystallization, we find that quantities such us total energy and volume are constant for $t>50 \mathrm{ps}$. Thus, the first $50 \mathrm{ps}$ of the simulation are discarded and the rest of the simulation is used for data acquisition. Moreover, the correlation time (obtained from the intermediate scattering function) in bulk water simulations using the SPC/E model at $T=300 \mathrm{~K}$ and $\rho=1.0 \mathrm{~g} / \mathrm{cm}^{3}$ is approximately $3 \mathrm{ps} 88$. Thus, our simulations appear long enough to avoid effects of the equilibration process on our calculations.

\section{RESULTS: HYDROPHOBIC PLATES}

\section{A. Capillary evaporation: effect of pressure}

In this section, we describe the effects of pressure $P$ and plate-plate separation $d$ on the phase behavior of water confined between hydrophobic plates at $T=300 \mathrm{~K}$. To consistently define $d$, we use the distance between the planes containing the hydrogen $(H)$ atoms on the inner surfaces of two hydrophilic plates. In the case of hydrophobic plates, where no hydrogen atoms are present, the same planes (where the $H$ atoms would hypothetically be located) are used to define $d$. As previously noted, the plane containing the $H$ atoms is located at a distance $0.033 \mathrm{~nm}$ from the plane containing the oxygen atoms of the silanol groups.

Figure 2]summarizes the results of our MD simulations 
for $d \geq 0.5 \mathrm{~nm}$. Two observations are most relevant from Fig. 2] (i) the threshold separation distance $d_{t h}$ required for capillary evaporation decreases with increasing $P$, and (ii) for $d \approx 0.6 \mathrm{~nm}$ a bilayer crystal is obtained at high $P$. Simulations for $d \leq 0.4 \mathrm{~nm}$ at $P=0.2 \mathrm{GPa}$ and $0 \mathrm{GPa}$ show that molecules are trapped interstitially between the plate atoms. The water molecules remain in a plane and are not able to diffuse due to the lack of space between plates.

The decrease in $d_{t h}$ with increasing $P$ can be understood as follows. Equating the grand potential of the confined liquid and the confined vapor, for sufficiently large surfaces [35, 50],

$$
-P A d_{t h}+2 A \gamma_{w l} \approx-P^{*} A d_{t h}+2 A \gamma_{w v}
$$

whence [35, 50, 54],

$$
d_{t h} \approx \frac{2 \Delta \gamma}{\left(P-P^{*}\right)}
$$

In the above equations, $A$ is the plate surface area; $\gamma_{w l}$ and $\gamma_{w v}$ are the wall-liquid and wall-vapor interfacial tensions $\left(\Delta \gamma \equiv \gamma_{w l}-\gamma_{w v}\right) ; P$ is the bulk pressure; and $P^{*}$ is the equilibrium vapor pressure at the given temperature. It follows from Eq. (2) that $d_{t h}$ decreases with increasing $P$, as manifest in Fig. 2. For an incompressible liquid, $P-P^{*}=\rho_{l}\left(\mu_{l}-\mu_{v}\right)$, and, therefore [21, 35],

$$
d_{t h} \approx \frac{2 \Delta \gamma}{\rho_{l}\left(\mu_{l}-\mu_{v}\right)}
$$

where $\mu_{l}$ and $\mu_{v}$ denote the chemical potentials of the liquid at $P$ and of the vapor (and liquid) at $P^{*}$. In the above derivation we have assumed that the plate characteristic size, $\approx A^{1 / 2}$, is large enough so that one can neglect the finite lateral size of the confined region (i.e., $\left.d_{t h} / A^{1 / 2} \ll 1\right)[35]$.

A sequence of snapshots showing a typical dewetting process is given in Fig. 3 We observe that the density rapidly becomes rarified on a time scale of $\approx 10 \mathrm{ps}$, and then the solvent retreats on a somewhat longer time scale $(\approx 100 \mathrm{ps})$. At $P=0 \mathrm{GPa}$ and $d \leq 0.6 \mathrm{~nm}$, the vapor cavity is limited to the area of the plates, i.e. the water surrounding the plates remains in the liquid state [Fig. 3(d)]. However, at the lower pressure $P=-0.05 \mathrm{GPa}$ and $d \leq 1.2 \mathrm{~nm}$, the bubble originally formed between the plates expands to the bulk liquid, inducing cavitation of the whole system. In other words, a heterogeneous nucleation event occurs induced by the plates. For $P \leq-0.1 \mathrm{GPa}$ and $d \leq 1.6 \mathrm{~nm}$, we observe a simultaneous cavitation inside and outside of the confined space indicating that, for the present water model, the liquid phase is unstable for $P \leq-0.1 \mathrm{GPa}$. We note that when capillary evaporation occurs, no water molecule is observed between the plates. Thus, practically speaking, what we call 'vapor phase' is here a vacuum.

Observations consistent with this picture have been reported in measurements of the force between hydrophobically-coated mica surfaces immersed in water
[53]. Specifically, vapor cavities formed spontaneously when mica surfaces coated with a double-chain cationic fluorocarbon surfactant approached to distances between 1 and $4 \mathrm{~nm}$ [53]. However, cavities only formed after separation from contact when the mica surfaces were coated with a double-chain cationic hydrocarbon surfactant [53]. Both the separation at which cavities formed and the magnitude of the force between the hydrophobic surfaces were found to be very sensitive to the specific substance used to coat the mica surface [52, 53]. The measured width of the dewetting layer formed at an extended paraffin surface in water $(\approx 1.5 \mathrm{~nm})$ is also consistent with the present calculations [55].

Our results on capillary evaporation are in agreement with previous grand canonical ensemble Monte Carlo simulations of water using the SPC model [89]. That work shows that capillary evaporation between smooth planar hydrophobic walls occurs at walls separations $D \approx 1.27 \mathrm{~nm}$ and $D \approx 0.9 \mathrm{~nm}$ at $P=0 \mathrm{GPa}$ and $P \approx 0.1 \mathrm{GPa}$, respectively. These distances correspond, in our case, roughly to $d \approx 0.79 \mathrm{~nm}$ and $d \approx 0.42 \mathrm{~nm}$ (see Fig. 2).

The presence of a crystal at high $P$ has not been discussed so far in the context of capillary evaporation. However, there is considerable evidence from MD simulations showing that confined water may crystallize at high $P$ (see e.g. 90]). Koga et al. found crystallization in MD simulations of the TIP4P model at $T \approx 230-300 \mathrm{~K}$ and $P=0.5-1.0 \mathrm{GPa}$ [91, 92, 93]. The ice structure that we find is similar to that found in [91, 93. and resembles none of the structures of the existing ice phases in bulk water, nor those found in metallic or hydrophobic pores 94, 95, 96, 97]. Figure 4(a), a top view of the bilayer ice, clearly shows a hexagonal lattice, while Fig. 4(b) suggests that each layer is almost flat, and that the two layers are in registry.

To confirm that confined water crystallizes at high $P$ and $d \approx 0.6 \mathrm{~nm}$, we calculate the mean square displacement (MSD) parallel to the plates at $P=0.2 \mathrm{GPa}$ for different values of $d$. Figure [5(a) shows the MSD for a 400 ps simulation averaging over molecules (we do not average over starting times). For $d=0.4 \mathrm{~nm}$, we find that $\mathrm{MSD} \approx 0$ consistent with the earlier observation that molecules are trapped between the plate atoms. For $d=0.5,0.8$, and $1.0 \mathrm{~nm}$ the MSD increases monotonically with time and, for a fixed time, with $d$. However, the MSD for $d=0.6 \mathrm{~nm}$ shows a fast increase for $t \lesssim 50$ ps and then approaches an asymptotic value of $\approx 0.2 \mathrm{~nm}$. Snapshots of the system during the first $50 \mathrm{ps}$ show that the molecules in the confined space reorganize to form the crystal. For $50 \mathrm{ps} \lesssim t \lesssim 300 \mathrm{ps}$, the resulting ice shows defects that disappear with time. The transformation is almost over for $t \approx 300$ ps. In the absence of crystallization, one would expect the MSD at long times for $d=0.6 \mathrm{~nm}$ to fall between that corresponding to $d=0.5 \mathrm{~nm}$ and $d=0.8 \mathrm{~nm}$. Instead, the MSD curve for $d=0.6 \mathrm{~nm}$ shows a plateau, as for $d=0.4 \mathrm{~nm}$, after about $250 \mathrm{ps}$, consistent with the view that the system 
crystallizes.

The crystallization of confined water is also confirmed by structural properties such as the radial distribution function (RDF) parallel to the plates, $g_{x y}(r)$, and the probability density function to find a molecule located at $z$ between the plates, $P_{n}(z)$. Figure [5 (b) shows $g_{x y}(r)$ for the ice $(d=0.6 \mathrm{~nm})$ and for a bilayer liquid $(d=0.8 \mathrm{~nm})$. The oscillations in $g_{x y}(r)$ for $d=0.6 \mathrm{~nm}$ are a clear sign of crystallization. Such oscillations are not present in the liquid phase. We note that the slow decay of $g_{x y}(r)$ for large $r$ is due to the finite size of the plates. This finite size effect can be removed by taking the ratio of $g_{x y}(r)$ for the crystal to that of the liquid (see inset). The persistence of oscillations is then clear. Figure [5] (c) shows the $P_{n}(z)$ for the ice $(d=0.6 \mathrm{~nm})$ and for a comparable liquid $(d=0.8 \mathrm{~nm})$. While in both cases water molecules form two layers [98], the peaks of $P_{n}(z)$ are much larger at $d=0.6 \mathrm{~nm}$ than at $d=0.8 \mathrm{~nm}$. $P_{n}(z)$ was calculated from a histogram of water molecules in 78 slices into which the distance between plates was divided. It is defined so that $\int P_{n}(z) d z=1$.

We note that no spontaneous crystallization in bulk or confined water has been reported in MD simulations using the SPC/E model. Crystallization using the TIP4P [99 and TIP5P models has been obtained 100]. In our case, we note that the location of water molecules belonging to each of the two ice layers is highly correlated with the plate structure, suggesting that the crystal we obtain is templated by the plate. Such substrate-templated crystallization of interfacial water has been observed on hydrophilic surfaces 78]. Figure 6(a) is a schematic diagram showing both the hexagons formed by the water oxygen atoms and the atoms at the plate surface. The atoms at the plate surface are arranged in tetrahedra pointing either into or out of the page of the figure. These two kinds of tetrahedra alternate, forming rings composed of six tetrahedra [see also Fig. 11(a)]. Two rules determine the structure of a given hexagon of the ice layers: (i) a water oxygen atom is located on top of each tetrahedron pointing into the page of the figure; and (ii) a water oxygen atom is located on top of the center of the hexagonal rings formed by the tetrahedra at the plate surface. As a result, the ice layers are formed by perfect hexagons with an $O O$ distance of $0.29 \mathrm{~nm}$. Interestingly, this is the same distance separating the two ice layers [see Fig. [5](c)]. Thus, the $O O$ distance between nearest and next nearest neighbors are $0.29 \mathrm{~nm}$ and $\sqrt{3} \times 0.29 \mathrm{~nm}=0.49 \mathrm{~nm}$, respectively. These values are in agreement with the location of the first two maxima of $g_{x y}(r)$ [see Fig. [5] (b)] which are located at $\approx 0.29 \mathrm{~nm}$ and $\approx 0.51 \mathrm{~nm}$, respectively.

The bilayer ice is fully hydrogen-bonded and each molecule has four HBs, as in ordinary ice. HBs occur either between molecules in the same ice layer or between both layers (i.e., there are no HB pointing toward the plates). The two water layers are linked by HBs where a molecule of one layer shares one of its $H$ atoms with the nearest oxygen atom of the other layer [see Figure 6(b) and (c)]. We note that the $\mathrm{HOH}$ angle in the $\mathrm{SPC} / \mathrm{E}$ model is $109.47^{\circ}$, i.e., the tetrahedral angle. However, in the bilayer ice structure, the $O O O$ angles (formed between three nearest neighbors) are either $90^{\circ}$ or $120^{\circ}$. Therefore, in general, the $O O$ directions (between nearest neighbors) differ slightly from the $O H$ directions of a given water molecule.

In refs. 101, 102, it was found that TIP4P water within carbon nanotubes can crystallize forming a tube of square, pentagonal, or hexagonal ice, depending on the nanotube radius. The structure of the carbon nanotube hexagonal ice seems to be the same as that found in the present work. Interestingly, the same requirements [that the confinement geometry allows: (i) next-nearest oxygen atoms to be located roughly at the bulk O-O distance $(\approx 0.28 \mathrm{~nm}$ ) [102]; and (ii) water molecules to have four hydrogen bonds] are necessary for ice formation in our case and in the carbon nanotubes.

Simulations of water between crystalline infinite walls using the TIP5P model [90, 103, 104|, and between smooth infinite walls using the TIP5P 105] and TIP4P models 92] also show the formation of crystals at $T=$ $300 \mathrm{~K}$. Both monolayer and tri-layer ices have been reported [104, 105]. As indicated in Fig. 2] our simulations at $P=0.2 \mathrm{GPa}$ show no sign of monolayer ice at small $d$ nor of crystallization to other solids at larger $d$ (corresponding to $n$-layer crystals, $n \geq 3$ ). Small variations in $d(\lesssim 0.1 \mathrm{~nm})$ were found to have a profound effect on the appearance and disappearance of confined ices [92, 103, 104]. In Fig. 2] the values of $d$ simulated at a given $P$ are separated by $\Delta d \geq 0.1 \mathrm{~nm}$. Thus, it is possible (but unlikely) that these ices can be found for confined $\mathrm{SPC} / \mathrm{E}$ at $T \approx 300 \mathrm{~K}$ when using a smaller $\Delta d$.

Figure 2 also shows a schematic confined phase diagram superimposed on the simulation data points. The bilayer crystal and liquid phases are indicated, together with an estimate of the dewetting transition line. At $T=300 \mathrm{~K}$, we find that the dewetting transition line extends down to $d=0.5 \mathrm{~nm}$, suggesting that it merges with the region (defined by $d \lesssim 0.4 \mathrm{~nm}$ ) for which molecules gets trapped between the plate atoms. It is interesting to consider whether for a higher $T$ the dewetting transition line might end in a critical point. The relationship between the dewetting locus and water's ordinary vapor-liquid critical point will be investigated in future studies at higher temperatures. It is also possible that the bilayer crystal region in Fig. 2 expands down to the dewetting transition line (see red dotted line in the figure). In this case, there would be a triple point where the vapor phase coexists with the liquid and crystal phases. Our simulation at $P=0.05 \mathrm{GPa}$ and $d=0.6 \mathrm{~nm}$ does not show the presence of a crystalline structure. Instead, we observe a competition between a disordered (liquid) structure and bubble formation. At 600 ps a bubble occupies $25 \%$ of the confined space, and at $1 \mathrm{~ns}$ we find that $90 \%$ of the confined space is dewetted. With the size of the plate area that we simulate, it is not possible to observe simultaneously the vapor, liquid, and crystal 
phases [only approximately five hexagons per side can be clearly observed in Fig. [(a)] . A precise investigation of the existence (or lack thereof) of a triple point requires larger plate surface areas than we have used in this work.

\section{B. Water structure: effect of pressure}

To study the effects of pressure on the structure of water we fix $d=1.6 \mathrm{~nm}$. This separation is large enough so that water properties in the middle region between the plates are essentially those of bulk water [46].

Figure 7 shows the average water density between the plates at each pressure, $\rho(P)$. For comparison, we show also the density of bulk water reported in 88 . We define $\rho(P)=\langle n(t)\rangle \times m_{H 2 O} / V_{\text {conf }}$ where $m_{H 2 O}$ is the mass of a water molecule and $\langle n(t)\rangle$ is the average number of molecules in the confined space between the walls. When dealing with confined systems, defining the accessible volume for the confined liquid is not unique. We estimate $V_{\text {conf }}$ in two ways: by the formal dimensions of the confined space, based on the defined $d$, i.e., $V_{\text {conf }}=1.6 \times 3.217 \times 3.215 \mathrm{~nm}^{3}$; and by an effective available volume $V_{\text {conf }}=d_{\text {eff }} \times 3.217 \times 3.215 \mathrm{~nm}^{3}$ where $d_{e f f}$ is an effective plate-plate distance. If $P_{n}(z)$ is the probability density to find an $O$ atom in a slab parallel to the plates located at $z\left(\int P_{n}(z) d z=1\right)$, then $d_{\text {eff }}$ is defined such that $P_{n}\left( \pm d_{e f f} / 2\right)=0.5$ [see Fig. 8(a)]. The first approach to the calculation of $V_{\text {conf }}$ underestimates $\rho(P)$ because the formal confined volume extends practically to the plate surface (i.e., the plane where the $H$ atoms would be located), and thus includes space not accessible in practice to water molecules. The second (effective) definition of $V_{\text {conf }}$ overestimates $\rho(P)$ because it leaves out from the calculation a portion of volume that is in fact accessible to water molecules.

$\rho(P)$ in the confined space is always smaller than the bulk density for $P \lesssim 0.06 \mathrm{GPa}$, independently of the method used to calculate $V_{\text {conf }}$. For $P>0.06 \mathrm{GPa}$ the two estimates of $V_{\text {conf }}$ produce drastically different results. One expects that at high $P$ the water density in the confined space approaches that of the bulk water. It can be seen from Fig. [7 that the formal definition of $V_{\text {conf }}$, using the $d$-value defined a priori, leads to a more reasonable high- $P$ trend. For all cases, one finds that the slope of $\rho(P)$ is always greater than that of bulk water indicating greater compressibility between the plates. To confirm this, we interpolate $\rho(P)$ in Fig. 7 with a second order polynomial, and calculate the corresponding derivative with respect to $\mathrm{P}$. The resulting values for the compressibility, $\kappa_{T}$, at $P=0 \mathrm{GPa}$ and $T=300 \mathrm{~K}$ are: $\kappa_{T} 0.47 \mathrm{GPa}^{-1}$, for bulk water; and $\kappa_{T} 1.47 \mathrm{GPa}^{-1}$ (formal volume) and $\kappa_{T} 1.70 \mathrm{GPa}^{-1}$ (effective volume), for confined water. The experimental value of the compressibility at these conditions is $\kappa_{T} 0.45 \mathrm{GPa}^{-1}$ 106, , in good agreement with the simulations.

Figure 8(a) shows the density profile $\rho_{\text {slab }}(z)$, i.e. the local density in a slab parallel to the plates, located at $z$.
It was calculated using 39 slabs of width $0.0411 \mathrm{~nm}$, with an area equal to the plates area. $\rho_{\text {slab }}(z)$ resembles the number density at normal $T$ and $P$ reported in Ref. [46]. Figure 8 (a) indicates that the main effect of increasing $P$ is to push water molecules toward the plates. Accordingly, as $P$ increases, $\rho_{\text {slab }}(z)$ increases for $z \approx 0$ and the maxima at $z \pm 0.51 \mathrm{~nm}$ for $P=-0.05 \mathrm{GPa}$ shift to $z \pm 0.63 \mathrm{~nm}$ at $P=0.2 \mathrm{GPa}$. Moreover, the maxima and minima of $\rho_{\text {slab }}(z)$ are more pronounced at $P=0.2 \mathrm{GPa}$ than at $P=-0.05 \mathrm{GPa}$, as molecules increasingly sample 'harder' plates at high $P$. We obtain the same conclusions when looking at the probability density function for the $\mathrm{H}$ atoms.

To analyze the local packing and hydrogen bonding, we calculate the average coordination number $C N(z)$ and the order parameter $q(z)$ used to characterize the local tetrahedral order in water [107, 108]. Averages are taken over slabs of width $0.0411 \mathrm{~nm}$ centered at different $z$. We define $C N(z)$ as the number of neighbor oxygens within a distance $\leq 0.32 \mathrm{~nm}$ (the first minimum of the radial distribution function at $\rho=0.984 \mathrm{~g} / \mathrm{cm}^{3}$ and $T=284 \mathrm{~K}$ [109]) from a central $O$ atom. $q(z)$ is defined as [107]

$$
q \equiv 1-\frac{3}{8} \sum_{j=1}^{3} \sum_{k=j+1}^{4}\left(\cos \psi_{j k}+\frac{1}{3}\right)^{2}
$$

where $\psi_{j k}$ is the angle formed by the lines joining the oxygen atom of a given molecule and those of nearest neighbors $j$ and $k(\leq 4)$. In this work, we also include the oxygen $(O)$ atoms in the plates when considering the $O$ nearest neighbors $j$ and $k$.

Figure 8 (b) shows $C N(z)$ for different values of $P$. At all $P$, we find that $C N(Z)$ for $z \approx 0$ is close to 4 as is the case for bulk water. The slight increase of $C N(z)$ with $P$ at $z=0$ can be due to the fact that we are using a fixed cutoff of $0.32 \mathrm{~nm}$ to calculate $C N$ (while the first minimum of the radial distribution function depends slightly on $P$ ). We observe that $C N(Z)$ decreases as one approaches the plates. However, right next to the plates we find sharp maxima. Moreover, such maxima increase with $P$ as water molecules are pushed toward the plates [see Fig. 8 (a)]. When the $O$ atoms in the plates are not considered in the calculation of $C N(z)$, we find a distribution similar to that of 46. In this case, $C N(z) \approx 2.5$ next to the plates, indicating that molecules in contact with the surfaces lose on average $1.5-2$ water neighbors.

Figure 8(c) shows $q(z)$ for different values of $P$. At all $P$, we find that $q(z) \approx 0.62$ at $z=0$, close to the value $0.60-0.65$ for bulk water at $0.85 \leq \rho \leq 1.15 \mathrm{~g} / \mathrm{cm}^{3}$ and $T=300 \mathrm{~K}$ [107] (indicated in the figure). The peaks of $C N(z)$ next to the plates are accompanied by the rapid decrease of $q(z)$ approaching the interface. At the plates, $q(z) \approx 0.5$, and this value does not change with $P$, that is, the local tetrahedrality is not apparently affected by $P$ for molecules in contact with the surfaces.

To study the orientational structure, we follow [46] and compute the distribution of angles $\theta_{h b}$ between the four 
HB vectors of water molecules and the inward pointing normal to the plates. Each molecule is associated with four $\mathrm{HB}$ vectors: these four vectors point tetrahedrally outwards from the oxygen Lennard-Jones site, and such that two of them join the $O$ site to the same molecule's $H$ atoms. The normalized distribution $P\left(\theta_{h b}\right)$ in the proximity of the plates is shown in Fig. 9 for different values of $P$. $P\left(\theta_{h b}\right)$ shows maxima at $\theta_{h b}=70^{\circ}$ and $180^{\circ}$ indicating a preferred orientation for a water molecule with one HB vector pointing toward the nearest plate. These results, and a $P\left(\theta_{h b}\right)$ calculated in slabs at different distances from the plates (not shown), are in agreement with the results obtained in ref. [46] in constant volume MD simulations. Interestingly, we find that when $P$ increases, despite changes in $\rho_{\text {slab }}(z), P\left(\theta_{h b}\right)$ barely changes, as is evident in Fig. 9. In particular, molecules next to the plates preserve their distinct orientational order under pressure. This is in agreement with the noted invariance of $q(z) \approx 0.5$ next to the surfaces for all $P$.

The radial distribution function (RDF) for the water molecule $O$ atom and the $O$ atom at the surface of the plate, $g_{O^{\prime} O}(r)$, is shown in Fig. 10. At all $P, g_{O^{\prime} O}(r)$ has maxima at $0.32,0.60$, and $0.77 \mathrm{~nm}$. For comparison, the first maximum of the bulk water RDF at $\rho=0.984 \mathrm{~g} / \mathrm{cm}^{3}$ and $T=284.5 \mathrm{~K}[109]$ is at $0.28 \mathrm{~nm}$. Thus, water oxygen atoms are located farther from plate surface oxygen atoms than from other water molecule oxygen atoms, as expected for hydrophobic surface atoms. The increase of $P$ does not shift the location of the maxima and minima of $g_{O^{\prime} O}(r)$, but changes their relative heights. As $P$ increases, more molecules are found in the confined space and, at the same time, more molecules shift from interstitial to coordination shells. Thus, the confined liquid becomes more structured upon compression.

In summary, the effect of pressure on the structure of water confined between hydrophobic plates is to enhance water structure, pushing the water molecules toward the plates. The orientation of water molecules next to the plates is not sensitive to compression, as indicated by Fig. 9

\section{RESULTS: HYDROPHILIC PLATES}

Over the range of conditions investigated here $(T=$ $300 \mathrm{~K}, P=-0.05,0.05$, and $0.2 \mathrm{GPa}$, and in each case $d=0.6,1.0$, and $1.6 \mathrm{~nm}$ ), we find that the confined water remains in the liquid state, and shows no sign of capillary evaporation or crystallization. In agreement with the hydrophobic plate simulations, we find that for $P \leq-0.1 \mathrm{GPa}$, the whole system cavitates; i.e., liquid water is unstable. Experimentally, this would manifest itself as loss of cohesion and the appearance of a macroscopic vapor phase; computationally, it becomes impossible to maintain tensions in excess of $0.1 \mathrm{GPa}$ and the system volume grows uncontrollably due to the appearance of the vapor phase. However, even in this case we find no capillary evaporation between hydrophilic plates.
In other words, liquid water fills the confined space, and the hydrophilic plates induce wetting even when there is bulk cavitation around the plates.

\section{A. Water structure: effect of pressure}

As previously done for the case of the hydrophobic plates, we chose a distance between plates of $d=1.6 \mathrm{~nm}$. The average density in the confined space between hydrophilic plates is shown in Fig. 11 As for the hydrophobic plates case, we define $\rho(P)=\langle n(t)\rangle \times m_{H 2 O} / V_{\text {conf }}$ and use both $d=1.6 \mathrm{~nm}$ and $P_{n}\left( \pm d_{\text {eff }}\right)=0.5$ to compute $V_{\text {conf }}$. In contrast to the hydrophobic case, both methods now lead to very similar $\rho(P)$ values. The underestimated (formal volume) and overestimated (effective volume) values of $\rho(P)$ are very close to each other and bracket those for bulk water. Interestingly, Fig. 11 shows that the slopes of $\rho(P)$ for confined water (using both volume definitions) are almost the same as that of bulk water. This suggests that the compressibility of water confined by hydrophilic plates is the same as that of bulk water. In fact, we calculate the compressibility as in Sec. IIIB for the case of water confined by hydrophobic plates. We find that, using either the formal volume or the effective volume, the compressibility of water confined by hydrophilic plates is $\kappa_{T} \approx 0.52 \mathrm{GPa}^{-1}$, close to the value $\kappa_{T} \approx 0.47 \mathrm{GPa}^{-1}$ of bulk water.

Figure 12 shows $\rho_{\text {slab }}(z), C N(z)$, and $q(z)$ for water confined between hydrophilic plates at different values of $P$. The three quantities are insensitive to $P$ over the range of conditions investigated here. Thus, the strong attraction exerted by the walls on liquid water is not measurably perturbed by the additional forces associated with compression. The two maxima of $\rho_{s l a b}(z)$ in Fig. 12 (a) clearly indicate the presence of two water layers next to the plates. We note that Fig. [12(a) is very similar to Fig. 3 of Ref. 110] for the TIP4P water model. From Fig. 12(b) and (c), we find that both $C N(z)$ and $q(z)$ in the presence of hydrophilic plates decrease to zero very sharply at the plate surfaces. Moreover, a comparison of Fig. 8(c) and [12 (c) shows that in the absence of the hydroxylated groups on the plates, the values of $q(z)$ start to decrease at larger distances from the walls than in the case of hydrophilic plates. The resulting profiles in Fig. 12 (b) and (c) are almost flat for the region between the plates meaning that the values of $C N(z)$ and $q(z)$ next to the plates barely change with respect to their bulk water values at $z=0$. These results suggest that water molecules far from the plates have similar local environment (in terms of tetrahedral order and number of nearest-neighbors) as those next to the plates (when including the plate surface oxygen atoms). For the molecules next to the plates, the $O-H$ atoms on the plate surface act as 'virtual' water molecules providing extra HBs to the real water molecules. Again, the slight change in $C N(z)$ at $z \approx 0$ is probably due to the fact that we use a fixed cutoff when calculating the coordination 
number.

Figure 13 shows $P\left(\theta_{h b}\right)$ corresponding to a slab next to the plates of width $0.1 \mathrm{~nm} . P\left(\theta_{h b}\right)$ is in agreement with the corresponding distribution computed in ref. [110] for the TIP4P water model and is complementary to the distribution found in the case of hydrophobic plates (see Fig. 91. The presence of polarity on the plate allows water molecules to form HBs with atoms on the plate. The resulting $P\left(\theta_{h b}\right)$ increases abruptly for $\theta_{h b}<20^{\circ}$ and has a sharp peak at $\theta_{h b} \approx 110^{\circ}$. As already found in 110 for the TIP4P water model, these maxima can be explained by the presence of multiple HBs that a water molecule can have with $O H$ groups associated with the plate (see Fig. 14 in [110]). Ultrafast electron crystallography measurements of water confined between hydrophilic walls [78 also show that water molecules interact at two sites of the substrate.

The RDFs between the plate oxygen $\left(O^{\prime}\right)$ or hydrogen $\left(H^{\prime}\right)$ atoms and the water molecule oxygen atoms are shown in Fig. 14. These distributions do not change with $P$, confirming the insensitivity of confined water structure between hydrophilic plates to changes in external pressure. Figure 14 is in agreement with Figs. 11 and 12 of Ref. [110], which were obtained for the TIP4P water model.

\section{SUMMARY}

We have presented results from MD simulations of water confined between nanoscale (hydrophobic or hydrophilic) plates at $T=300 \mathrm{~K}$ and for a range of values of pressure $P(-0.15 \mathrm{GPa} \leq P \leq 0.2 \mathrm{GPa})$ and plateplate separation $d(0.4 \mathrm{~nm} \leq d \leq 1.6 \mathrm{~nm})$.

In the case of hydrophobic plates, a phase diagram in the $P-d$ plane summarizing the MD results is presented, and three phases (liquid, vapor, and bilayer ice) are identified. At low $P$ we find capillary evaporation. The distance at which this drying occurs decreases with increasing $P$. Furthermore, the transition line in the $P-d$ phase diagram separating the vapor and liquid phases is followed to small values of $d$, below which water molecules become individually trapped and immobilized by the surface atoms. It is possible that at higher $T$, this line ends in a critical point at high $d$ (corresponding to the liquid-vapor critical point of bulk water).

The bilayer ice is composed of two layers of hexagons in registry along the surface normal. The resulting ice is similar to that found in Ref. [91]. This ice is found at $P \geq 0.1 \mathrm{GPa}$ and $d \approx 0.6 \mathrm{~nm}$, but only in a narrow range of $d$. The region corresponding to ice in the $P-d$ phase diagram might be connected with the vapor-liquid transition line for larger plates, corresponding to a triple point. However, the plates we simulate are small, and we cannot observe simultaneously the liquid, vapor and crystal phases. Further investigation is required on this point. We also note that at $T=300 \mathrm{~K}$ and in the presence of nanoscale plates, we do not find indications of a monolayer ice, like that observed in MD simulations using the TIP4P and TIP5P potentials and infinite walls [103].

When simulating water confined by hydrophilic (hydroxylated silica) plates, we find that the confined water remains in the liquid phase at all $P$ and $d$ studied. Moreover, even at $P$ where we observe cavitation in the bulk water, the confined water is in the liquid phase. In other words, the hydrophilic plates induce wetting in the confined space (a phenomenon reminiscent of capillary condensation). This result suggests that one way to stabilize liquid water under tension may be by hydrophilic confinement. Experiments of water confined between a hydrophobic and a hydrophilic surface (Janus interface) [77, suggest a similar physical picture, where the hydrophobic surface encourages water to dewet, while the hydrophilic surface constrains water to be present.

We also study the effect of $P$ on the hydration of both hydrophobic and hydrophilic plates. We focus on the water molecule distribution, average coordination number and local tetrahedral order parameter along the direction normal to the plates. In the case of hydrophobic plates, all of these quantities indicate that as $P$ increases, water molecules are pushed toward the plates while the liquid becomes more structured. However, the water molecule orientation next to the plates shows no dependence on $P$. As previously reported in ref. 46], molecules have on average one HB pointing to the plate. Strikingly, for the case of hydrophilic plates, we find no change in the liquid structure with $P$ in the range $-0.1 \mathrm{GPa}$ to $0.2 \mathrm{GPa}$.

The strong differences observed in the behavior of water confined between hydrophilic and hydrophobic plates leads to the interesting question of what the thermodynamic and structural properties of water are when it is confined between heterogeneous plates (e.g., hydrophobic plates that have been partially hydroxylated). Results addressing this question will be reported in a subsequent report.

\section{Acknowledgments}

We thank F. W. Starr for providing the basic SPC/E code for water using the reaction field method, M.S. Shell for fruitful discussions related to the Ewald sum method, and F. Sciortino for enlightening discussions related to the simulation details. We also thank A. Luzar for very useful comments on the manuscript. PJR gratefully acknowledges support by the R. A. Welch Foundation (F0019). PGD and PJR gratefully acknowledge the support of the National Science Foundation (Collaborative Research in Chemistry Grant Nos. CHE0404699 and CHE0404695). 
[1] B. Hille, Ionic Channels of Excitable Membranes (Sinauer, Sunderlan, MA, 1992).

[2] O. Beckstein, P.C. Biggin, and M.S.P. Sansom, J. Phys. Chem. B 105, 12902 (2001); O. Beckstein and M.S.P. Sansom, Proc. Natl. Acad. Sci. U.S.A. 100, 7063 (2003).

[3] E. Tajkhorshid, P. Nollert, M.Ø. Jensen, L.J.W. Miercke, J. O'Connel, R.M. Stroud, and K. Schulten, Science 296, 525 (2002); M.Ø. Jensen, E. Tajkhorshid, and K. Schulten, Biophys. J. 85, 2884 (2003).

[4] K. Murata, K. Mitsuoka, T. Hirai, T. Walz, P. Agre, J.B. Heymann, A. Engel, and Y. Fujiyoshi, Nature (London) 407, 599 (2000).

[5] D. Fu, A. Libson, L.J.W. Miercke, C. Weitzman, P. Nollert, J. Krucinski, and R.M. Stroud, Science 290, 481 (2000).

[6] H. Sui, B.-G. Han, J.K. Lee, P. Wallan, and B.K. Jap, Nature (London) 414, 872 (2001).

[7] Structure and Dynamics of Mambranes, Vols. 1A and 1B, in Handbook of Biological Physics, Edited by R. Lipowsky and E. Sackmann (Elsevier, Amsterdam, 1995).

[8] J. Faeder and B.M. Ladanyi, J. Phys. Chem. B 104, 1033, (2000); J. Faeder and B. M. Ladanyi, J. Phys. Chem. B 109, 6732 (2005); M.R. Harpham, B.M. Ladanyi, N.E. Levinger, and K.W. Herwig, J. Chem. Phys. 121, 7855 (2004).

[9] P.G. Debenedetti, Metastable Liquids, Concepts and Principles, (Princeton University Press, Princeton, 1996).

[10] R.M. Barrer, Zeolites and Clay Minerals as Sorbents and Molecular Sieves (Academic, London, 1978).

[11] A.D. Darhuber and S.M. Troian, Annu. Rev. Fluid Mech. 37, 425 (2005).

[12] Corrosion Mechanisms in Theory and Practice (2nd ed., P. Marcus, ed., Marcel Dekker, New York, 2002).

[13] A. Lafuma and D. Quere, Nature Materials 2, 457 (2003).

[14] L. R. Pratt and A. Pohorille, Chem. Rev. 102, 2671 (2002).

[15] M. Freemantle, Chem. Eng. News 77, 27 (1999).

[16] D. Chandler, Nature 437, 640 (2005).

[17] C. Tanford, The Hydrophobic Effect: Formation of $\mathrm{Mi}$ celles and Biological Membranes (Wiley, New York, 1980), 2nd ed.

[18] A. Ben-Naim, Hydrophobic Interactions (Plenum, New York, 1980).

[19] N.T. Southall, K.A. Dill, and A.D.J. Haymet, J. Phys. Chem. B 106, 521 (2002).

[20] J. Israelachvili, Intermolecular and Surface Forces (Academic Press, London, 1991).

[21] K. Lum, D. Chandler, and J.D. Weeks, J. Phys. Chem. B 103, 4570 (1999).

[22] G. Hummer, S. Garde, A.E. García, A. Pohorille, and L.R. Pratt, Proc. Natl. Acad. Sci. USA 93, 8951 (1996).

[23] F.H. Stillinger, J. Sol. Chem. 2, 141 (1973).

[24] T. M. Truskett, P. G. Debenedetti, and S. Torquato, J. Chem. Phys. 114, 2401 (2001).

[25] A. B. Kolomeisky and B. Widom, Faraday Discuss. 112, 81 (1999).

[26] S. B. Zhu, G. W. Robinson, J. Chem. Phys. 94, 1403 (1990).
[27] M. Majumder, N. Chopra, R. Andrews, and B.J. Hinds, Nature 438, 44 (2005).

[28] G. Hummer, J. C. Rasaiah, and J. P. Noworyta, Nature 414, 188 (2001).

[29] S. Andreev, D. Reichman, and G. Hummer, J. Chem. Phys. 123, 194502 (2005).

[30] R. J. Hunter, Foundation of Colloid Science (Oxford University Press., Oxford U.K., 1987).

[31] G. Hummer, S. Garde, A.E. García, and L.R. Pratt, Chem. Phys. 258, 349 (2000).

[32] Y.-K. Cheng, W.-S. Sheu, and P. Rossky, Biophys. J. 76, 1734 (1999).

[33] R.R. Dogonadze, E. Kálmán, A.A. Kornyshev, and J. Ulstrup, Eds., The Chemical Physics of Solvation, ch. 11 (Elsevier, Amsterdam, 1985).

[34] A. Terfort, N. Bowden, and G.M. Whitesides, Nature (London) 386, 162 (1997).

[35] K. Lum and A. Luzar, Phys. Rev. E 56, 6283 (1997); A. Luzar and K. Leung, J. Chem. Phys. 113, 5836 (2000); K. Leung and A. Luzar, J. Chem. Phys. 113, 5845 (2000).

[36] K. Leung, A. Luzar, and D. Bratko, Phys. Rev. Lett. 90, 65502 (2003).

[37] J.S. Rowlinson and B. Widom, Molecular Theory of Capillarity (Oxford University Press, Oxford, 1982).

[38] S. Rajamani, T.M. Truskett, and S. Garde, Proc. Natl. Acad. Sci. USA 102, 9475 (2005).

[39] D.A. Zichi and P.J. Rossky, J. Chem. Phys. 83, 797 (1985).

[40] Q. Du, E. Freysz, and Y.R. Chen, Science 264, 826 (1994).

[41] X. Huang, C.J. Margulis, and B.J. Berne, J. Chem. Phys. B 107, 11742 (2003).

[42] A.C. Belch and M. Berkowitz, Chem. Phys. Lett. 113, 278 (1985).

[43] A. Wallqvist, J. Phys. Chem. 95, 8921 (1991).

[44] A. Wallqvist and B.J. Berne, J. Phys. Chem. 99, 2893 (1995).

[45] A. Wallquist and B.J. Berne, J. Phys. Chem. 99, 2885 (1995).

[46] C.Y. Lee, J.A. McCammon, and P.J. Rossky, J. Chem. Phys. 80, 4448 (1984).

[47] G.M. Torrie, P.G. Kusalik, and G.N. Patey, J. Chem. Phys. 89, 3285 (1988).

[48] A.A. Gardner and J.P. Valleau, J. Chem. Phys. 86, 4171 (1987).

[49] N. Choudhury and B. Montgomery Pettitt, J. Am. Chem. Soc. 127, 3556 (2005).

[50] J.L. Parker, P.M. Claesson, and P. Attard, J. Phys. Chem. 98, 8468 (1994).

[51] Y-H. Tsao, S.X. Yang, D.F. Evans, and H. Wennerström, Langmuir, 73154 (1991).

[52] R.M. Pashley, P.M. McGuiggan, and B.W. Ninham, Science 229, 1088 (1985).

[53] H.K. Christenson and P.M. Claesson, Science 239, 390 (1988).

[54] D.R. Bérard, P. Attard, and G.N. Patey, J. Chem. Phys. 98, 7236 (1993).

[55] T.R. Jensen et al., Phys. Rev. Lett. 90, 086101 (2003).

[56] X. Huang, C.J. Margulis, and B.J. Berne, Proc. Natl. Acad. Sci. USA 100, 11953 (2003). 
[57] X. Huang, R. Zhou, and B.J. Berne, J. Phys. Chem. B 109, 3546 (2005).

[58] J. Li et al., J. Chem. Phys. B 109, 13639 (2005).

[59] T. Koishi et al., Phys. Rev. Lett. 93, 185701 (2004); T. Koishi, K. Yasuoka, T. Ebisuzaki, S. Yoo, and X.C. Cheng, J. Chem. Phys. 123, 204707 (2005).

[60] M. C. Gordillo, G. Nagy, J. Mart, J. Chem. Phys. 123, 54707 (2005).

[61] N. Choudhury and B. Montgomery Pettitt, J. Chem. Phys. B 109, 6422 (2005).

[62] W. Kauzmann, Adv. Protein Chem. 14, 1 (1959).

[63] K.A. Dill, Biochemistry 29, 7133 (1990).

[64] A.R. Fersht, Structure and Mechanism in Protein Science (W.H. Freeman and Co., New York 1999).

[65] C.L. Brooks, J.N. Onuchic, and D.J. Wales, Science 293, 612 (2001); C.L. Brooks, M. Gruebele, J.N. Onuchic, and P.G. Wolynes, Proc. Natl. Acad. Sci. U.S.A. 95, 11037 (1998).

[66] C.M. Dobson, A. Sali, and M. Karplus, Angew. Chem., Int. Ed. Engl. 37, 868 (1998).

[67] P.L. Privalov and G.I. Makhatadze, J. Mol. Biol. 232, 660 (1993).

[68] B. Honig and A.-S. Yang, Protein Chem. 46, 27 (1995).

[69] J.M. Sturtevant, Proc. Natl. Acad. Sci. U.S.A. 74, 2236 (1977).

[70] D.H. Williams, M.S. Searle, J.P. Mackay, U. Gerhard, and R.A. Maplestone, Proc. Natl. Acad. Sci. U.S.A. 90, 1172 (1993).

[71] N. Froloff, A. Windemuth, and B. Honig, Protein Sci. 6, 1293 (1997).

[72] Y.-K. Cheng and P.J. Rossky, Nature 392, 696 (1998).

[73] R. Zhou, X. Huang, C.J. Margulis, and B.J. Berne, Science 305, 1605 (2004).

[74] P. Liu, X. Huang, R. Zhou, and B.J. Berne, Nature (in press).

[75] M.P. Stoykovich, M. Müller, S.O. Kim, H.H. Solak, E.W. Edwards, J.J. de Pablo, and P.F. Nealey, Science 308, 1442 (2005).

[76] J. Israelachvili and R. Pashley, Nature (London) 300, 341 (1982).

[77] X. Zhang, Y. Zhu, and S. Granick, Science 295, 663 (2002).

[78] C.-Y. Ruan et al., Science 304, 80 (2004).

[79] H.J.C. Berendsen, J.P.M. Postma, W.F. van Gunsteren, A. DiNola, and J.R. Haak, J. Phys. Chem. 81, 3684 (1984).

[80] H.J.C. Berendsen, J.R. Grigera and T.P. Stroatsma, J. Phys. Chem. 91, 6269 (1987).

[81] R.K. Iler, The Chemistry of Silica (Wiley, New York, 1979); C. Hammond, The basics of Crystallography and Diffraction (Oxford University Press, 2001), page 31.

[82] See also http://cst-www.nrl.navy.mil/lattice/struk/c9.html

[83] A.Y. Toukmaji and J.A. Board Jr., Comp. Phys. Comm. 95, 73 (1996).

[84] T.M. Nymand and P. Linse, J. Chem. Phys. 112, 6152 (2000).

[85] T. Werder, J. H. Walther, R. L. Jaffe, T. Halicioglu, and P. Koumoutsakos, , J. Phys. Chem. B 107, 1345 (2003).

[86] In this work the values of $P$ do not include corrections due to the cutoff $\left(r_{c}=0.79 \mathrm{~nm}\right)$ used when calculating the plate-water, plate-plate, and water-water LennardJones interactions. For the case of a Lennard-Jones (LJ) liquid, the correction to $P$ is given by $[\mathrm{M}$. P. Allen and
D. J. Tildesley, Computer simulation of liquids, Oxford University Press (2004)]

$$
\Delta P=\frac{32 \pi}{9} \frac{\epsilon \sigma^{12}}{r_{c}^{9}} \frac{N_{L J}^{2}}{V^{2}}-\frac{16 \pi}{3} \frac{\epsilon \sigma^{6}}{r_{c}^{3}} \frac{N_{L J}^{2}}{V^{2}}
$$

where $N_{L J}$ is the number of particles. We can estimate $\Delta P$ in our simulations by using this equation with $N_{L J}=3375+1348=4723$ (i.e., the number of oxygen atoms of the water molecules plus the number of atoms in the plates that have LJ interactions) and the values of $\epsilon$ and $\sigma$ from the SPC/E model. This gives, $\Delta P[G P a] \approx-821 / V^{2}\left([V]=\mathrm{nm}^{3}\right)$. Thus, depending on the volume of our simulations we get $-0.063 \mathrm{GPa}$ $<\Delta P<-0.047 \mathrm{GPa}$.

[87] D.C. Rapaport, The art of molecular dynamics simulations (Cambridge University Press, 1995).

[88] F. W. Starr, F. Sciortino, and H.E. Stanley, Phys. Rev. E 60, 6757 (1999). For a better comparison with our results, the values of $P$ taken from this work have been corrected so Lennard-Jones corrections are eliminated (see [86]).

[89] D. Bratko, R. A. Curtis, H. W. Blanch, and J. M. Prausnitz, J. Chem. Phys. 115, 3873 (2001).

[90] R. Zangi, J. Phys.: Condens. Matter 16, S5371 (2004).

[91] K. Koga, X.C. Zeng, and H. Tanaka, Phys. Rev. Lett. 79, 5262 (1997).

[92] K. Koga and H. Tanaka, J. Chem. Phys. 122, 104711 (2005).

[93] K. Koga, H. Tanaka, X. C. Zeng, Nature 408, 564 (2000).

[94] J.D. Porter and A.S. Zinn-Warner, Phys. Rev. Lett. 73, 2879 (1994).

[95] N. Materer et al., J. Phys. Chem. 99, 6267 (1995).

[96] K.M. Park, R. Kuroda, and T. Iwamoto, Angew. Chem. Int. Ed. Engl. 32, 884 (1997).

[97] M. Odelius, M. Bernasconi, and M. Parrinello, Phys. Rev. Lett. 78, 2855 (1997).

[98] The presence of the small third peak in Fig. 5. is due to crystal defects occurring during the ice formation (approximately $t<300 \mathrm{ps}$ ), which causes some diffusing molecules between the two ice layers during the transient crystallization to be included in the statistics. Furthermore, there are "border effects" due to the finite size of the plates. Thus, molecules at the boundaries of the plates (which do not belong to the crystal) might have been included in the statistics.

[99] M. Matsumoto, S. Saito, and I. Ohmine, Nature 416, 6879 (2002).

[100] M. Yamada, S. Mossa, H.E. Stanley, and F. Sciortino, Phys. Rev. Lett. 88, 195701 (2002).

[101] R. J. Mashl, S. Joseph, N. R. Aluru, and E. Jakobsson, Nano Letters 3, 589 (2003).

[102] K. Koga, G. T. Gao, H. Tanaka, and X. C. Zeng, Nature 412, 802 (2001).

[103] R. Zangi and A.E. Mark, Phys. Rev. Lett. 91, 025502 (2003).

[104] R. Zangi and A.E. Mark, J. Chem. Phys. 119, 1694 (2003).

[105] P. Kumar, S.V. Buldyrev, F.W. Starr, N. Giovambattista, and H.E. Stanley, Phys. Rev. E 72, 051503 (2005).

[106] R. J. Speedy and C. A. Angell, J. Chem. Phys. 65, 851 (1976); R. A. Fine and F. J. Milero, J. Chem. Phys. 59, 5529 (1973). 
[107] J.R. Errington and P.G. Debenedetti, Nature 409, 318 (2001).

[108] N. Giovambattista, P.G. Debenedetti, F. Sciortino, and H.E. Stanley, Phys. Rev. E 71, 061505 (2005).

[109] F. Sciortino, P. Gallo, P. Tartaglia, and S.-H. Chen, Phys. Rev. E 54, 6331 (1996).

[110] S.H. Lee and P.J. Rossky, J. Chem. Phys. 100, 3334 (1994).
TABLE I: Potential parameters for plate-water interactions (taken from Ref. 110]).

\begin{tabular}{cccc}
\hline \hline Atom Type $\epsilon[\mathrm{kJ} / \mathrm{mol}]^{(a)}$ & $\sigma[\mathrm{nm}]^{(a)}$ & Charge $[\mathrm{e}]^{(b)}$ \\
\hline$O$ & 0.6487 & 0.3154 & -0.71 \\
$S i$ & 0.5336 & 0.3795 & 0.31 \\
$H$ & - & - & 0.40 \\
\hline \hline
\end{tabular}

(a) Lennard-Jones parameter for plate-water $O$ atom interactions (hydrophobic and hydrophilic plates).

(b) Charges on $\mathrm{Si}-\mathrm{O}-\mathrm{H}$ groups (hydrophilic plates).

TABLE II: Simulation times for the different kinds of plates and plate separation $d$.

\begin{tabular}{ccc}
\hline \hline Plate & $d[\mathrm{~nm}]$ & Simulation time $[\mathrm{ps}]$ \\
\hline hydrophobic $>0.8$ & 200 \\
hydrophobic $\leq 0.8^{(a)}$ & $400^{(a)}$ \\
hydrophilic $\leq 1.6$ & 200 \\
\hline \hline
\end{tabular}

(a) For hydrophobic plates with $d=0.6 \mathrm{~nm}$ and $P=0.05 \mathrm{GPa}$, the simulation time is $1 \mathrm{~ns}$. 


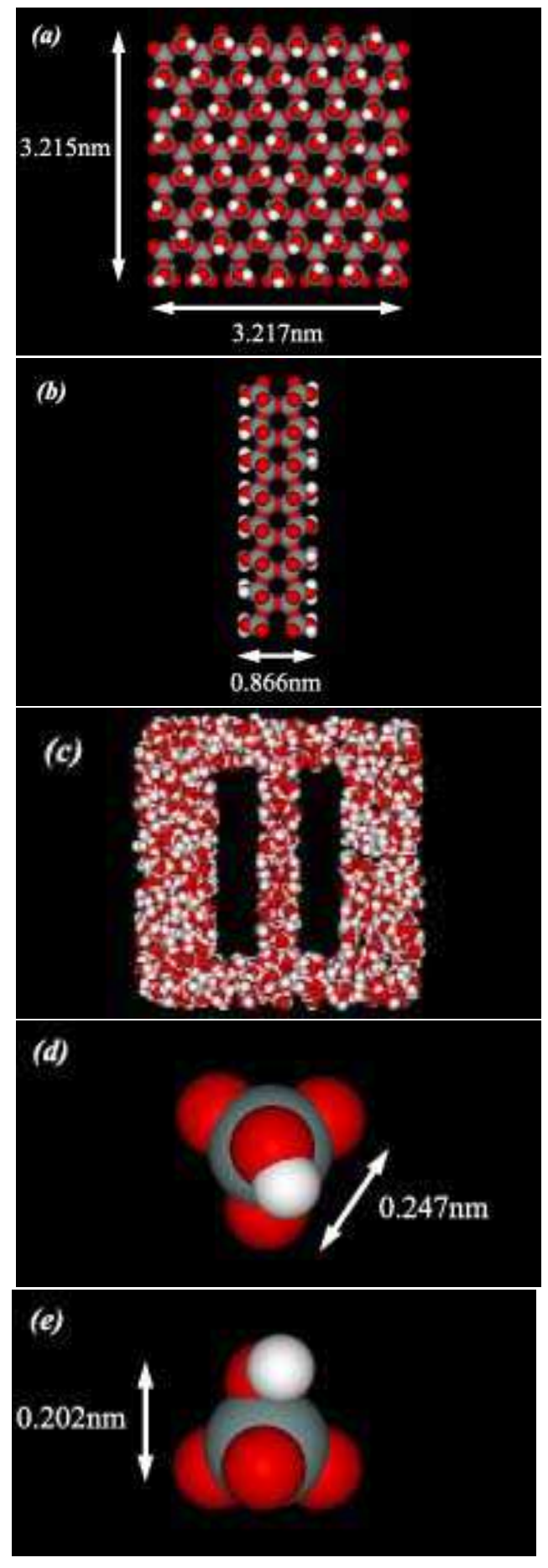

FIG. 1: (Color online) (a) Front and (b) lateral view of the fully hydroxylated silica plate [(1.1.1) octahedral face of cristobalite], one of the two kinds of plates used in this study. White, red, and gray spheres represent hydrogen, oxygen, and silicon atoms, respectively. (c) $y-z$ cross section showing the space available to the water molecules. (d) Top and (e) lateral view of the surface $\mathrm{SiO}_{4}$ unit, in which the surface oxygen atom is hydroxylated. 


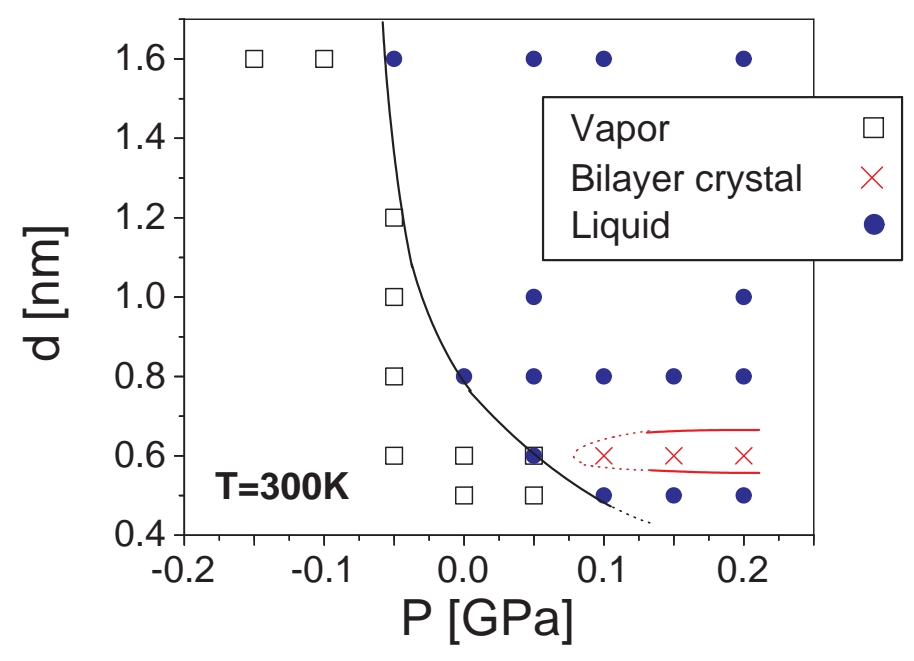

FIG. 2: (Color online) Phase behavior of confined water as a function of pressure and separation between nanoscale hydrophobic plates. The temperature is fixed at $T=300 \mathrm{~K}$. Filled circles indicate liquid state points. Empty squares indicate vapor state points at which capillary evaporation occurs (i.e., we observe no water molecule between plates). For $d \approx 0.6 \mathrm{~nm}$, a bilayer crystal is formed as indicated by red $\times$ symbols. At $d=0.6 \mathrm{~nm}$ and $P=0.05 \mathrm{GPa}$, fluctuations between liquid and vapor states are observed for at least $1 \mathrm{~ns}$. Continuous lines suggest a schematic phase diagram based on the simulation results. In the figure, the dashed red line corresponds to the assumption that the crystal region is not connected with the liquid-vapor transition line, i.e. that there is no 'triple point' between the liquid, the vapor, and the bilayer crystal. The black dotted line is the extrapolation of the liquid-vapor transition line into the $d \leq 0.4 \mathrm{~nm}$ region, where no liquid can be simulated due to the small distance between plates. 


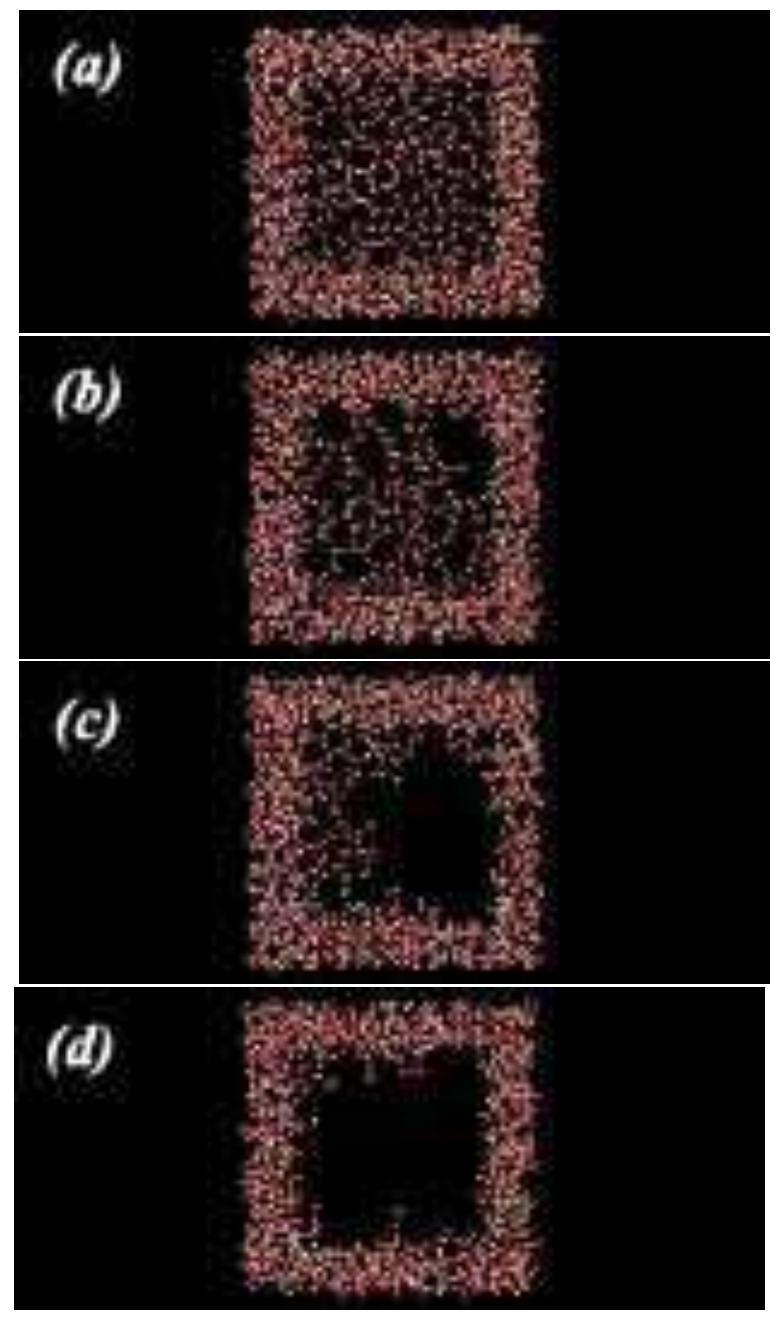

FIG. 3: (Color online) Sequence of time-separated snapshots showing capillary evaporation between hydrophobic plates at $P=0 \mathrm{GPa}$ and $d=0.6 \mathrm{~nm}$. Times correspond to (a) 18, (b) 40, (c) 135 , and (d) 239 ps. We only show water molecules in the slab corresponding to the space between plates (i.e., the plates themselves have been omitted for clarity). The square cavity evident at 239 ps corresponds to the whole area of the plates. 


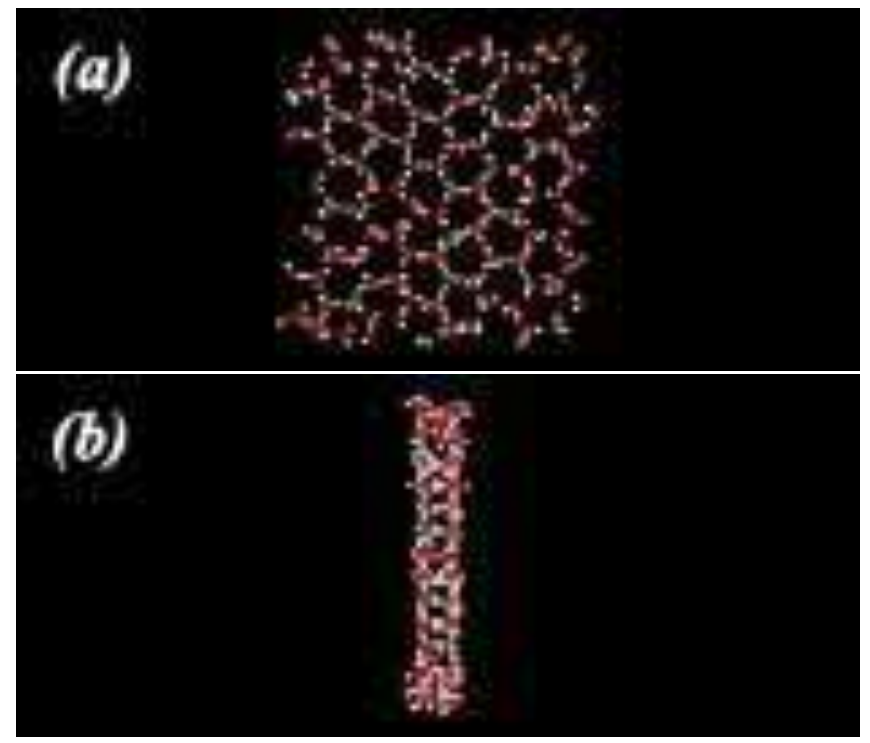

FIG. 4: (Color online) (a) Front and (b) lateral snapshots of the bilayer ice formed between hydrophobic plates at $P=$ $0.2 \mathrm{GPa}$ and $d=0.6 \mathrm{~nm}$. 

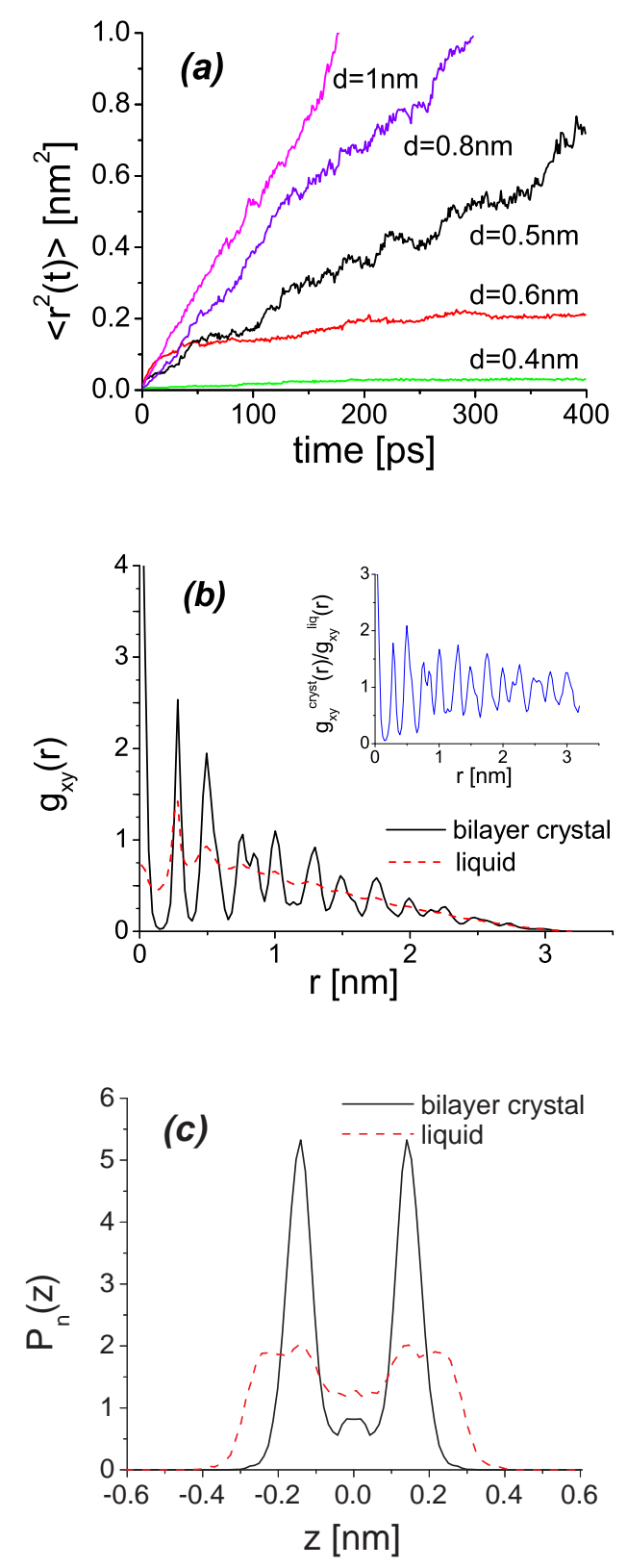

FIG. 5: (Color online) (a) Lateral mean square displacement (MSD) as a function of time at $P=0.2 \mathrm{GPa}$ and different plate-plate separations $d$. Hydrophobic plates. At $d=0.6 \mathrm{~nm}$ the confined water crystallizes and the MSD for long times is smaller than for $d=0.5,0.8$ and $1.0 \mathrm{~nm}$ (where the system is in the liquid phase). For comparison, we also show the results for $d=0.4 \mathrm{~nm}$ where water molecules are trapped between the plate atoms due to the small value of $d$. (b) Radial distribution function (RDF) parallel to the plates, $g_{x y}(r)$, for the crystal $(d=0.6 \mathrm{~nm})$ and liquid $(d=0.8 \mathrm{~nm})$ phases. Inset: ratio of the crystal RDF to that of the liquid. (c) Probability density function associated with the distribution of water molecules between the plates $\left(\int P_{n}(z) d z=1\right)$. 

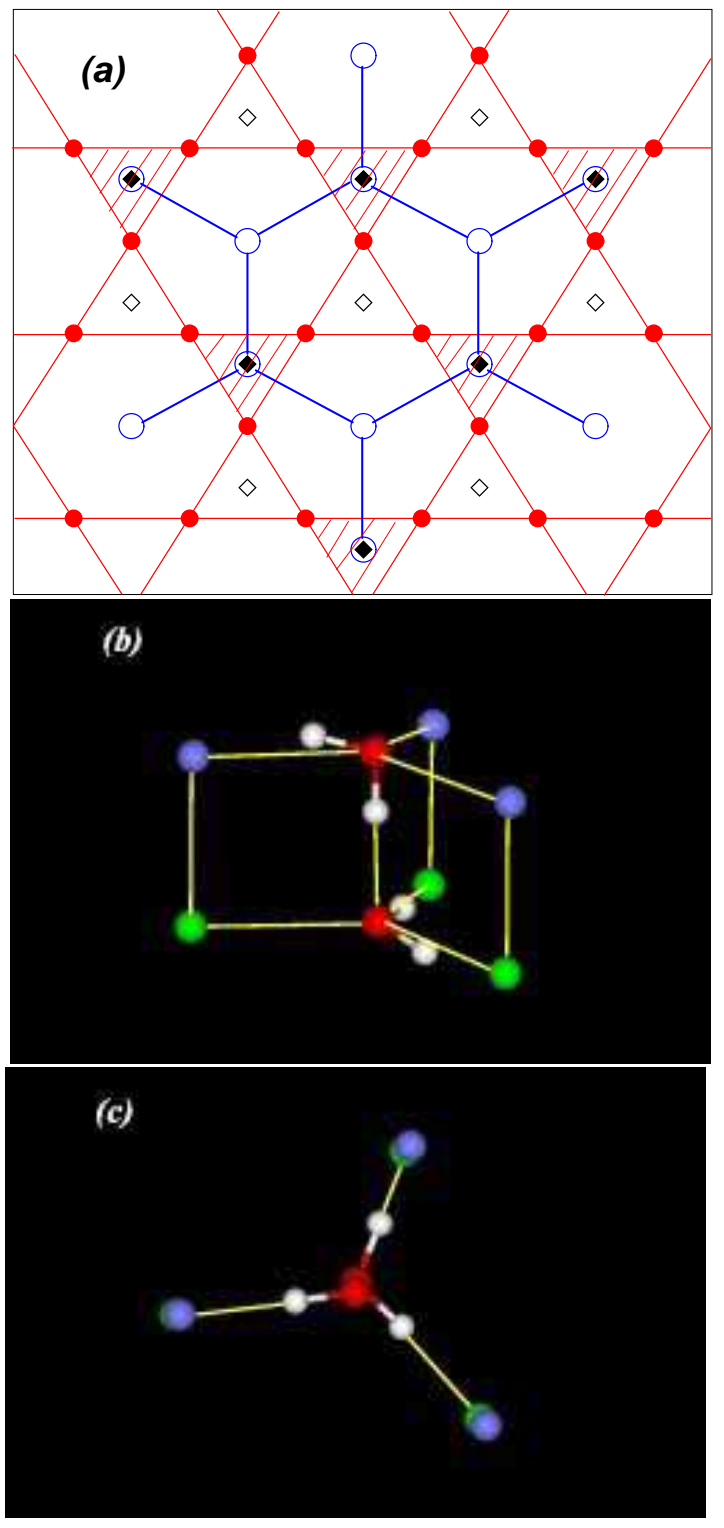

FIG. 6: (Color online) (a) Schematic diagram showing the location of water molecules in the bilayer ice phase relative to the position of the hydrophobic plate surface atoms. Red filled circles represent 'oxygen' atoms belonging to a single plane on the plate surface. The next layers of 'oxygen' atoms, above and below this plane, are represented by empty and filled black diamonds, respectively. The atoms at the plate surface are arranged in tetrahedra pointing either into or out of the page of the figure [cf. Fig. 1(d),(e)]. Tetrahedra pointing into the page correspond to the cross-hatched triangles centered on the filled diamonds, while those pointing out of the page correspond to triangles centered on the empty diamonds. Water oxygen atoms are represented by empty circles; a typical hexagon is indicated by blue lines. Lateral (b) and top (b) view of molecules belonging to the bilayer ice, showing the hydrogen bonds. We show only two water molecules with the corresponding hydrogen atoms (red and white spheres). These molecules belong to different layers of the ice and their corresponding nearest neighbors are represented by blue and green spheres. Yellow lines are a guide to the eye showing the lattice characterizing the bilayer ice. 


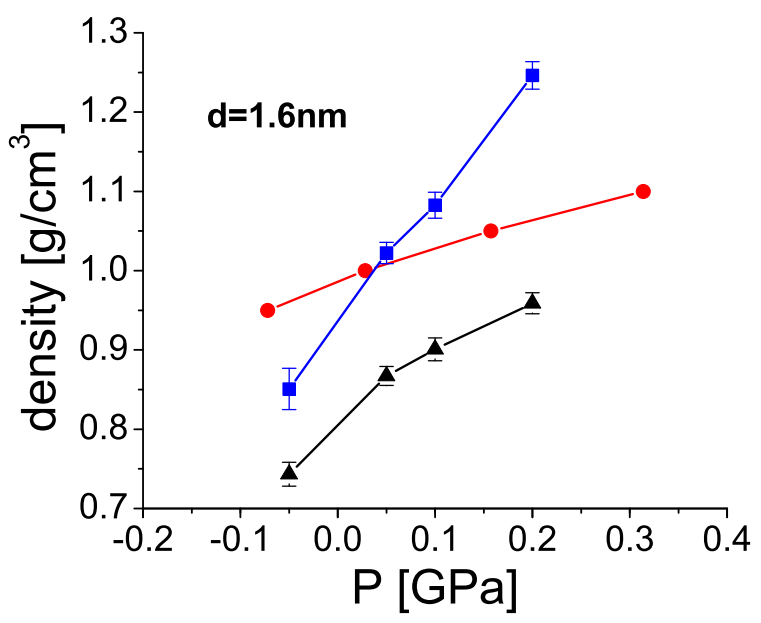

FIG. 7: (Color online) Average density $\rho(P)$ for different values of $P$ in the confined space between hydrophobic plates, and in bulk water. Circles correspond to the values of $\rho(P)$ for bulk water taken from ref. [88]. For confined water, we show two estimates of $\rho(P)$ corresponding to different definitions of the confined volume. Squares and triangles correspond to $\rho(P)$ calculations when using the 'effective' and 'formal' definition of volume, respectively (see Sect. IIIB for details). 

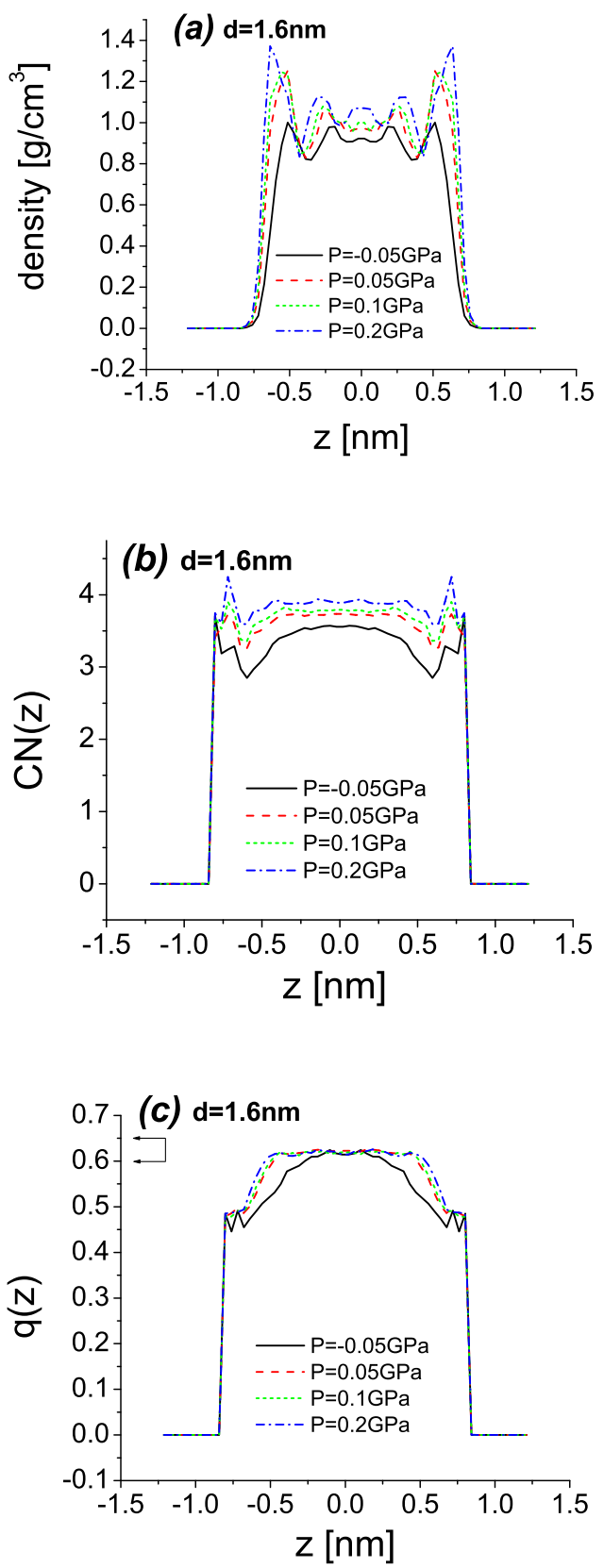

FIG. 8: (Color online) (a) Density profile, $\rho_{\text {slab }}(z)$ (i.e., the local density in a slab of width $0.0411 \mathrm{~nm}$, parallel to the plates, located at $z$ ); (b) average coordination number $C N(z)$; and (c) order parameter $q(z)$. Arrows in (c) indicate the range of $q$ values for bulk water at $T=300 \mathrm{~K}$ and $0.85 \mathrm{~g} / \mathrm{cm}^{3} \leq$ $\rho \leq 1.15 \mathrm{~g} / \mathrm{cm}^{3}$ [107]. Hydrophobic plates. 


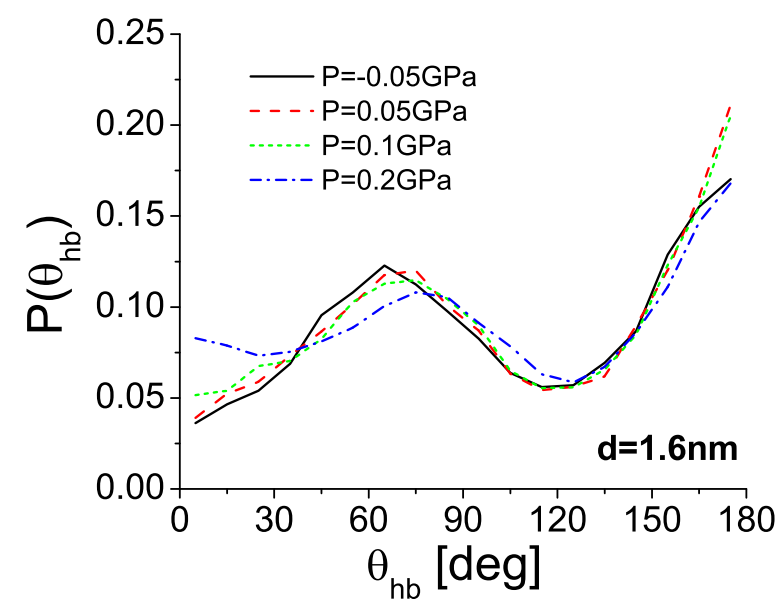

FIG. 9: (Color online) Normalized distribution $P\left(\theta_{h b}\right)$ of angles $\theta_{h b}$ between the hydrogen bond vectors of water molecules and the inward pointing normal to the plates. Average is performed over molecules at a distance $\leq 0.2 \mathrm{~nm}$ from the plates. Hydrophobic plates.

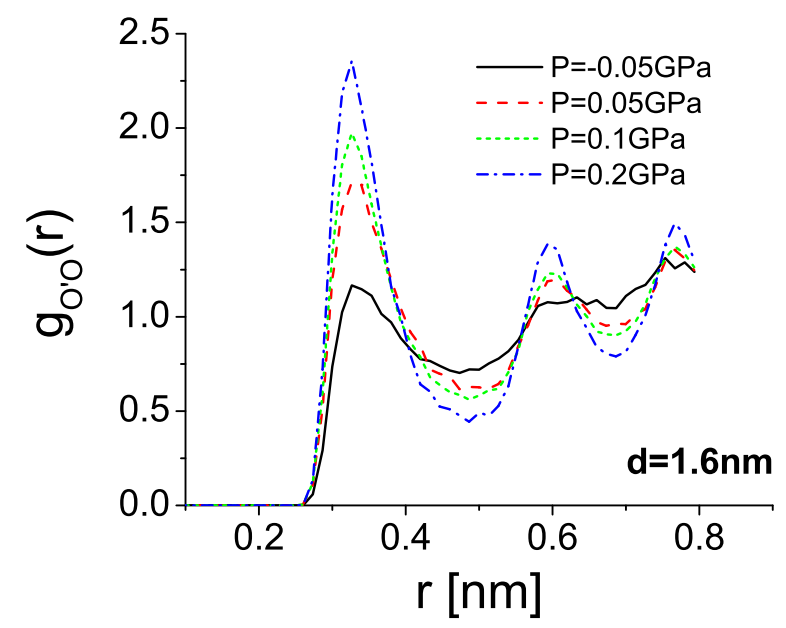

FIG. 10: (Color online) Radial distribution function for the water molecule $O$ and plate surface $O$ atoms. Hydrophobic plates. 


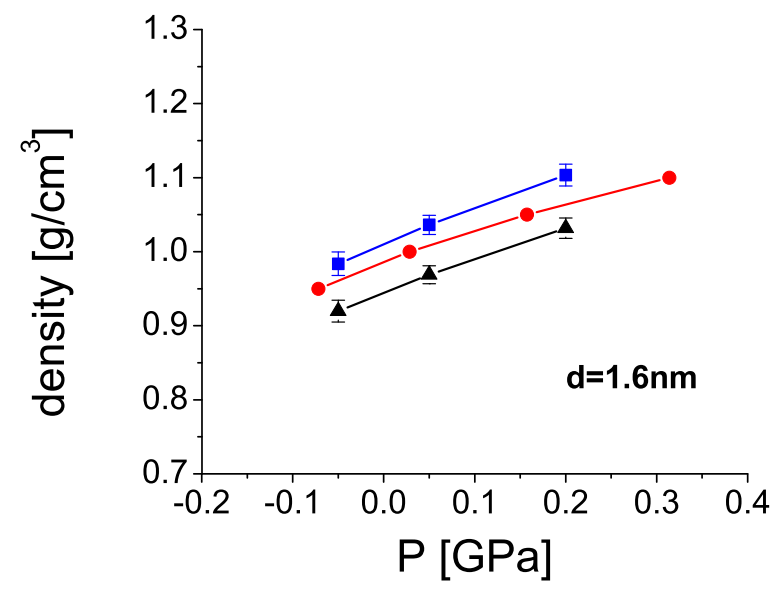

FIG. 11: (Color online) Density of water between hydrophilic plates compared to that of bulk water. Symbols and explanation are the same as in Fig. [7] 

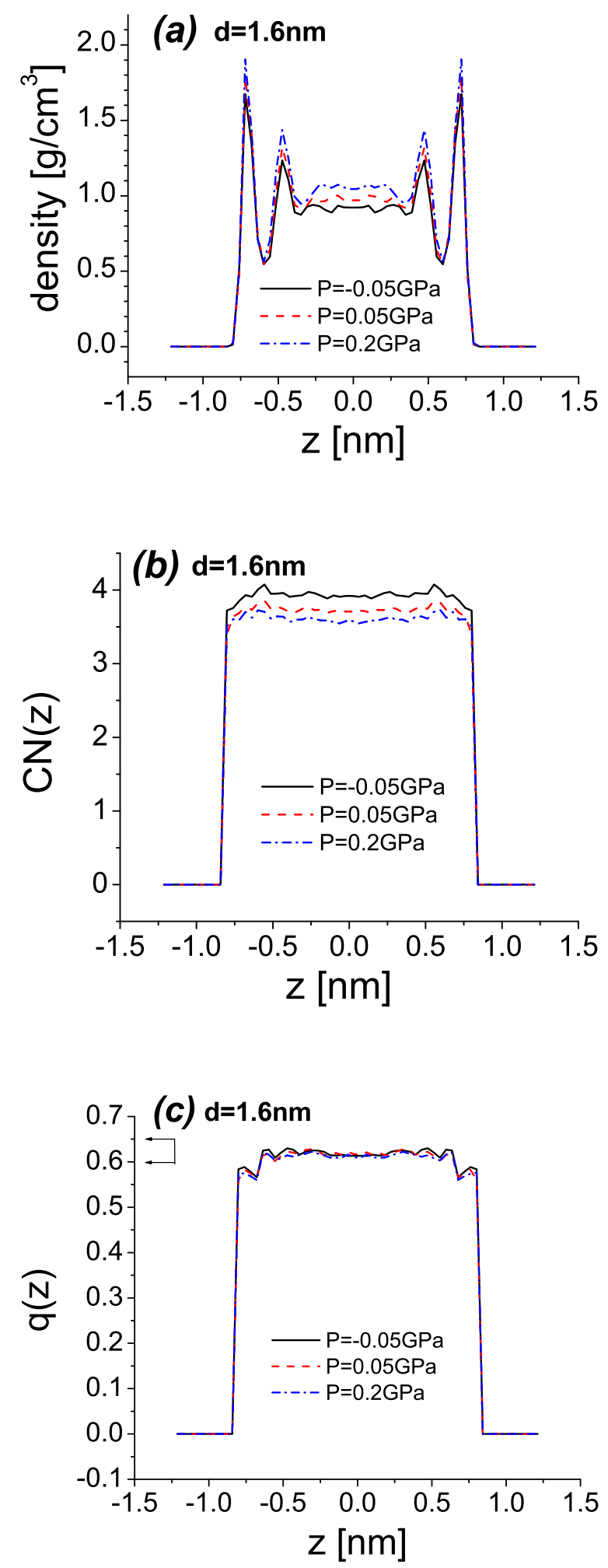

FIG. 12: (Color online) Same as Fig. 8 but for the case of hydrophilic plates. 


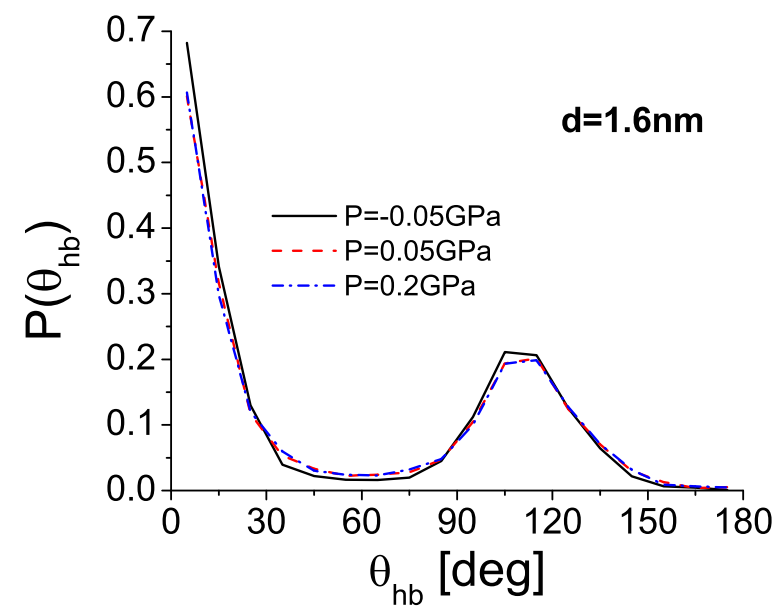

FIG. 13: (Color online) Normalized distribution $P\left(\theta_{h b}\right)$ of angles $\theta_{h b}$ between the hydrogen bond vectors of water molecules and the normal direction to the plates, for molecules at a distance $\leq 0.1 \mathrm{~nm}$ from the plates ( $c f$ Fig. 9). Hydrophilic plates. 

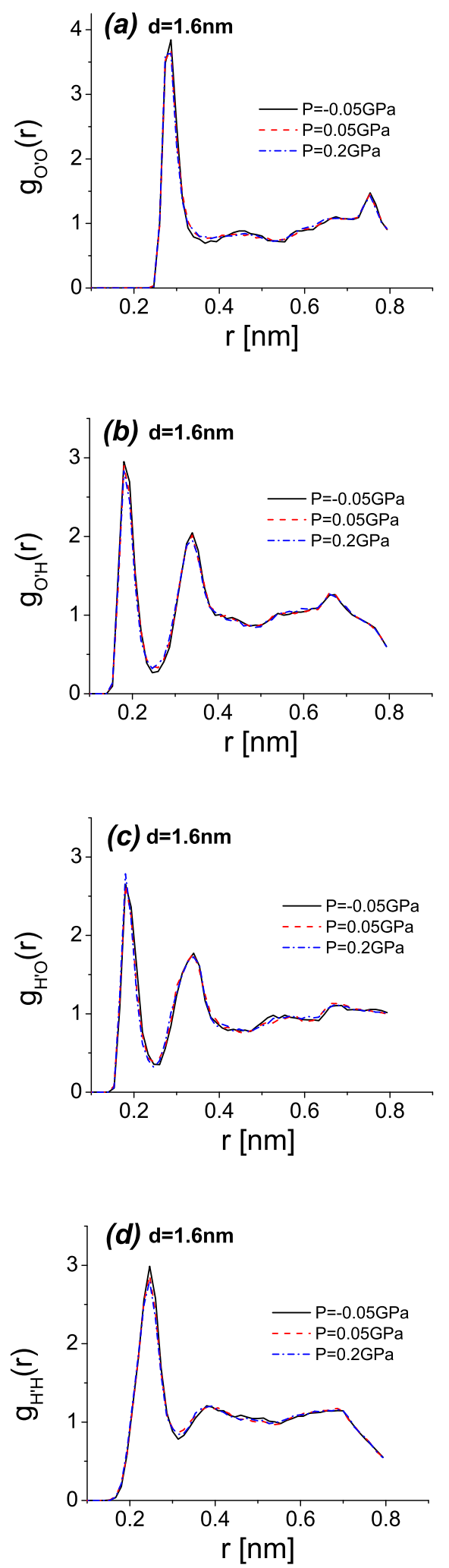

FIG. 14: (Color online) Radial distribution functions for the plate oxygen atoms $\left(O^{\prime}\right)$, and (a) the water oxygen and (b) water hydrogen atoms. (c)(d) Same as (a), (b) for the plate hydrogen atoms $\left(H^{\prime}\right)$. Hydrophilic plates. 